\title{
REDUCTION OF SEXTUPOLE DISTORTION BY SHUFFLING MAGNETS IN SMALL GROUPS ${ }^{\dagger}$
}

\author{
R. L. Gluckstern* \\ and
}

S. Ohnuma

\section{Summary}

A method is given for reducing the most troublesome sextupole harmonics in a ring by measuring the sextupole field in groups of magnets, and ordering them according to a predetermined prescription. The predicted result is a decrease in sextupole related distortions by a factor $\sqrt{ } 2 /(\mathrm{J}+1)^{\frac{1}{2}}$ where $\mathrm{J}$ magnets, covering one or more betatron periods, are measured at one time. Simulations performed for typical SSC lattices confirm the expected improvements.

Department of Physics and Astronomy, University of Maryland.

\footnotetext{
iSubmitted to the 1985 Particle Accelerator Conference, Vancouver, Canada, May 13-16, 1985.
} 
R.L. Gluckstern

Department of Physics and Astronomy

University of Maryland

College Park, Maryland 20742

and

s. Ohnuma

Fermi National Accelerator Laboratory*

P.0. Box 500, Batavia, Illinois 60510

\section{Summary}

A method is given for reducing the most troublesome sextupole harmonics in a ring by measuring the sextupole field in groups of magnets, and ordering them according to a predetermined prescription. The predicted result is a decrease in sextupole related distortions by a factor $[2 /(\mathrm{J}+1)]^{1 / 2}$ where $\mathrm{J}$ magnets, covering one or more betatron periods, are measured at one time. Simulations performed for typlcal SSC lattices confirm the expected improvements.

\section{Introduction}

The presence of unavoldable normal and skew sextupole errors in the dipoles of a superconducting ring are known to cause nonlinear osctllations in the beam size, and a resultant decrease in the dynamic aperture of the ring. Although the growth is not completely described by one or two "resonances", the harmonic description of the effect is useful in pointing out which regions of the harmonic spectrum are most troublesome. Such an analysi in first order in the sextupole amplitudes leads to the conclusion that beam size oscillation due to the $n$th harmonic of the sextupole error, $\varepsilon_{\mathrm{n}}$, are proportional to $\varepsilon_{\mathrm{n}}$ and inver sely proportional to the resonance denominators $|v-n|$ and $|3 v-n|$, where the $x$ and $y$ tunes, $v_{x}$ and $v_{y}$ are taken to be equal to $v$.

If all sextupole errors are known, it is concelvable that the order of the magnets could be chosen 80 that all harmonics in broad bands around $n=v$ and $n=$ $3 v$ could be made oufficlently small, thus reducing the beam dize osc1llations. Such "shuffling" 1s Impractical, however, since it requires measurements on all magnets before any can be positloned. For this reason, we propose an alternate scheme for measuring and positioning $\mathrm{J}$ magnets at a time, which 18 capable of reducing the expected value of all harmonics in the troublesome band, and therefore the beam atze osclllatlons, by a factor of order $\mathrm{J}^{-1 / 2}$.

\section{Description of Shuffiling Scheme}

For our analysis, we will consider a regular latt1ce consisting of $\mathrm{MJ}$ magnet $\mathrm{s}$, where $M$ is an integer near the tune $v$, and where $J$ magnet $s$ cover an integral number of magnet focusing periods. After measuring the first group of $J$ aagnets, we will place them in an order to be specified later, correlated wth the olze of the sextupole error. The next group of $J$ nagnets are then measured and placed in an order which is ant1-correlated with that in the first group, 1.e., the magnet wth the $j^{\text {th }}$ lowe pole error in the econd group 18 placed in the position corresponding to that of the jth highest (most positive) sextupole error in the first group. The process is then continued unt1l all magnets have been measured and positioned. Aside from statistical fluctuations in the size of the $f$ th highest sextupole error, we then have a sextupole error which repeats with a sign change every $J$ magnets. (Note that the parameters $\beta_{x}$ and $\beta_{y}$ repeat every J magnets.) Thus, we have now created systemat1c sextupole harmonics of order $M / 2,3 M / 2,5 M / 2$, etc. All other harmonics have been reduced in size because the width of the distr1bution of the $j$ th $h i g h e s t$ sextupole error is reduced from that of the total distribution by a factor of order $\mathrm{J}^{-1 / 2}$.

\section{Analysis of Shuffiling Scheme}

The atandard action-angle treatment of the third integer resonances ${ }^{1}$ leads to five driving terms in the Hamiltonian, each of which can be represented as a am of harmonics ${ }^{2}$. The form of the five coefficlents is lliustrated by a typical $n^{\text {th }}$ harmonic coefficient written in phase amplitude form as

$$
B_{n} e^{1 \beta_{n}}=(1 / 16 \pi) \sum_{m=1}^{M} \sum_{j=1}^{J} S_{m j}\left(\beta_{x}\right)^{3 / 2} e^{1 n \theta_{m j}+1 Q_{j}}
$$

Here $S_{m f}$ is the integrated strength of the $f^{\text {th }}$ sextupole in the $\mathrm{m}^{\text {th }}$ group of magnets and the amplitude functions $\beta_{x}$ and $B_{y}$ depend only on $j$ because of the perlodiclty of the lattice. The phase $Q_{f}$ reflects the difference between the phase $v \theta$ and the phase $\int \mathrm{ds} / \mathrm{B}$ within a cell and 18 therefore al 80 independent of $\mathrm{m}$. The independent varlable $\theta$ increases by $2 \pi$ in one revolution, and $\theta_{m j}$ is given by

$$
\theta_{m j}=\theta_{j}+\left(\frac{m-1}{M}\right) 2 \pi
$$

because of our measurement and placement scheme.

If we now perform the am over first in Eq. (1), ve have

$$
F_{j} \equiv \sum_{m=1}^{M} S_{n j} e^{2 \pi i n m / M}
$$

In order to proceed further, we now evaluate $\left\langle F_{f}\right\rangle$ and $\left\langle P_{j} F_{j}\right\rangle$, statistical averages over the distribution of sextupole errors. If we ssame a distribution of sextupole errors, $p(s)$, symmetric around $s=0$, the distribution of the $f^{\text {th }}$ highest $[(J+1-j)$ th lowe st] sextupole error $1 \mathrm{~s}$ given by

*Operated by the Universities Research Association, Inc. under contract with the U.S. Department of Energy. 


$$
\begin{gathered}
P_{j}(s) d s=\frac{J !}{(J-j) !(j-1) !} \\
{\left[\int_{-\infty}^{s} d x p(x)\right]^{J-j}\left[\int_{s}^{\infty} d x p(x)\right]^{j-1} p(s) d s}
\end{gathered}
$$

with

$$
P_{J+1-j}(B)=P_{j}(-B)
$$

One then finds, assuming $M$ is even,

$$
\begin{aligned}
&\left\langle F_{j}\right\rangle= \prod_{\ell=1}^{J} \int_{-\infty}^{\infty} d s_{\ell} P_{j}\left((-1)^{\ell} s_{\ell}\right) \sum_{m=1}^{M} s_{m j} e^{2 \pi i m m / M} \\
&=\langle g(j)\rangle\left[\sum_{\substack{m=2 \\
\text { even }}}^{M} e^{2 \pi i n m / M}-\sum_{\substack{m=1 \\
\text { odd }}}^{M-1} e^{2 \pi i n m / M}\right],
\end{aligned}
$$

where

$$
\langle s(j)\rangle \equiv \int_{-\infty}^{\infty} P_{j}(s) \text { sds }=-\langle s(J+1-j)\rangle
$$

It is now clear that the bracket in Eq. (6) vanishes for all $n$ except $n=r M / 2$ where $r 18$ an odd 1nteger. We then obtain

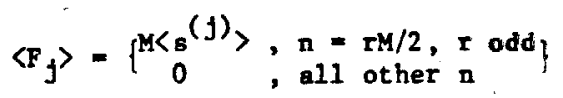

In a similar way, we find

$$
\left\langle F_{f^{*}} F_{j}^{*}\right\rangle=\left\langle F_{j}\right\rangle\left\langle F_{j}^{*}\right\rangle+M_{j j^{\prime}}
$$

where

$$
g_{j j^{\prime}} \equiv\left\langle\left\langle_{8}(j)_{8}\left(j^{\prime}\right)\right\rangle-\left\langle g^{(j)}\right\rangle\left\langle g^{\left(j^{\prime}\right)}\right\rangle .\right.
$$

For the untform distribution of sextupole errors

$$
p(s)=\left\{\begin{array}{cc}
1 /(2 \Delta) \\
0
\end{array},\left|\begin{array}{l}
s \\
s
\end{array}\right|<\Delta, \Delta,\right.
$$

one finds

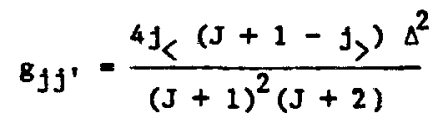

where $j_{\langle, j\rangle}$, are the maller, larger of $j, f^{\prime}$.

One now can write Eq. (1) in the fora

$$
\begin{aligned}
& \left\langle B_{n} e^{1 \beta_{n}}\right\rangle=
\end{aligned}
$$

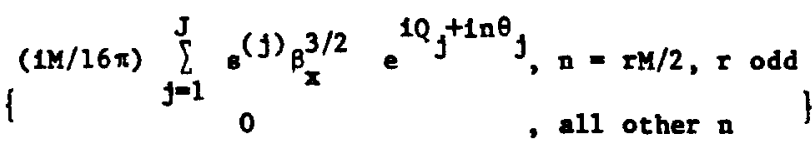

and, for all $\mathrm{n}$,

$$
\begin{gathered}
\left\langle B_{n}^{2}\right\rangle=\mid\left\langle\left. B_{n} e^{1 \beta_{n}}\right|^{2}+\right. \\
+\frac{M}{256 \pi^{2}} \sum_{j=1}^{j}\left(B_{\left.x j^{\prime} \theta_{x j^{\prime}}\right)^{3 / 2}} e^{1\left(Q_{j}-Q_{j^{\prime}}\right) \ln \left(\theta_{j}-\theta_{j^{\prime}}\right)}{\theta_{j j^{*}}}^{\text {(12) }}\right.
\end{gathered}
$$

We can only evaluate the aum over $f$ in $\left\langle B_{n}^{2}\right\rangle$ explicitly for a given lattice and ordering arrangement. However, we can estimate the oum by assuming constant $\beta$. and $Q_{f}$, and by neglecting the $j \neq f^{\prime}$ correlation terms In the expectation of some cancellation due to the phase variation of $a\left(\theta_{j}-\theta_{j}\right)$. In this way we find, for $n \neq r M / 2$,

$$
\left\langle B_{n}^{2}\right\rangle=\frac{\left\langle B_{x}^{3}\right\rangle}{256 \pi^{2}} \cdot \frac{M J \Delta^{2}}{3} \cdot \frac{2}{J+1}
$$

The first factor on the right comes frow Ohnuma's normalization of $B_{n}$, the second comes from the rms value of the sextupole error, and the third is the reduction factor coming frow our shuffing schewe, and correctly becomes unity for no shuffling ( $J=1$ magnet in each "group").

Although we have considered here only one of the five distortion parameters, our shuffling scheme will simultaneously reduce all five by approximately the same factor, aince the basic reduction comes from the reduced $\mathrm{rms}$ width of the distribution $\mathrm{P}_{j}(s)$.

It should al so be pointed out here that the specific result in Eq. (13) is valid only for a uniform distribution of sextupole errors. A closed form can also be obtalned for a parabollc dfstribution, bu: the algebra required $1 \mathrm{~s}$ much lengthier.

\section{Shuffling Within a Group}

The remaining calculation of $\left\langle B_{n} e^{1 \beta}\right\rangle$ in Eqs. (11) and (12) depends in detall on the cholce of the ordering scheme within a group, which is reflected in the values of $\theta_{j}$. Although the $\beta$ dependent factor in Eq. (12) and in one other driving term is $\beta_{x}^{3}$, the other three driving terms contaln the factor $\beta_{y}^{2}{ }_{x}$. The approximate constancy of $\beta_{x}+\beta_{y}$ implies that the sensitivity to variations of $\beta_{x}$ and $\beta_{y}$ is most acute for the driving terms with $\beta_{x}^{3}$. This aggests that magnets with the highest and lowest sextupole error should be placed where $\beta_{x}$ is malleat, that $1 B$, near the quadrupole which is defocussing in the $x$ direction. Also, it is desirable to alternate the signs of the sextupole errors in adjacent magnets $\infty$ that

$\left\langle B_{n} e^{1 B}\right.$, in Eq. (11) wll be algnificant only for large $r$. One possible arrangement between focussing agnets aight be

\section{defocussing quad}

$j=1$ dipole, highest $s$

$f=2$ dipole, 2 nd lowe st $s$

$j=3$ dipole, 3rd highest $s$

\section{focussing quad}

$$
\begin{gathered}
j=J-2 \text { dipole, } 3^{\text {rd }} \text { lowest } s \\
j=J-1 \text { dipole, } 2^{\text {nd }} \text { highest } s \\
j=J \text { dipole, lowest } s \\
\text { defocusing quad }
\end{gathered}
$$

Th1s echene concentrates the haraonic content into the 
Immediate vicinity of $r=\mathrm{J}$.

Simliar schemes are posstble if $J$ is an integral multiple of the number of magnets in a betatron osctllation period.

\section{Approximate Sum Rule}

In the approximation of constant $B_{x}^{3}$, 1t is easy to show from Eq. (1) that

$$
\sum_{n=1}^{M J} B_{n}^{2}=\frac{\left\langle B_{x}^{3}\right\rangle}{256 \pi^{2}} \sum_{m=1}^{M} \sum_{j=1}^{J} s_{m j}^{2}
$$

Thus, the and of the equares of the harmonfc ampl1tudes cannot be changed by shuffing. It 18 therefore apparent that any shuffling arrangement merely moves the sextupole harmonic content from one region of the spectrum to another. In our acheme we have depleted the harmonic content in all harmonics with $n \neq r M / 2$ at the expense of enhancing the ones wth $n=T M / 2$, which cause much less sextupole distortion. In particular, the shuffing sheme augested wthin a group concentrates the harmonic content into the ones close to $n$ $\mathrm{KJ} / 2$ which cause little multpole distortion.

\section{Two Parameter Shuffing}

There are cfrcumstances where one wants to reduce two independent error families at the same time. One such example occurs in magnets with uncorrelated but comarable normal and skew sextupole errors. Another would occur in any 2-1n-1 magnet assembly. A third example might be uncorrelated quadrupole and sextupole errors of comparable magnitude in a single dipole magnet.

We expect that the shuffling process outlined earlier would still work, but now one must order the errors into two parameter "bins". As a result the improvement factor is expected to be significantly reduced from that for one parameter shuffing.

\section{Numerical Regults}

Simulations have been performed for several SSC lattices, including insertions. In those cases where the number of magnets in a superperiod 18 not an 1ntegral multiple of twice the muber of magnet in a betatron perfod, a few of the "best" magnets are get aside for the unbalanced group, and the remaining ones distributed according to the original prescription. Four* of the five driving terms have been evaluated with ten Independent sets of random sextupoles, first with a truly random placement, and then with our aggested ordering. As a final figure of merit, ve obeain

$$
\lambda_{l}=\sum_{\mathrm{n}} \mathrm{B}_{\mathrm{nl}}^{\text {ordered }} / \int_{\mathrm{n}} \mathrm{B}_{\mathrm{n} \ell}^{\text {random }}
$$

for each of the four $(l=1-4)$ driving terms, where the aum over harmonics $\mathrm{n}$ is taken over the 100 harmonics centered at $M$ or $3 M$ as appropriate. Futhermore, the mumerator and denominator in Eq. (15) are averages over the ten Independent random sets of error 8 .

The following table Indicates the realts for uniform (U) or gaussian (G) error distributions for six (A - F) sample lattices.

Plgure of Merit

\begin{tabular}{|c|c|c|c|c|c|}
\hline Latt1ce & $\lambda_{1}$ & $\lambda_{2}$ & $\lambda_{3}$ & $\lambda_{4}$ & $\left(\frac{2}{J+1}\right)^{1 / 2}$ \\
\hline$A_{y}$ & .15 & .23 & .13 & .23 & .22 \\
\hline $\mathbf{A}_{G}$ & .11 & .21 & .19 & .28 & .22 \\
\hline $\mathrm{B}_{\mathrm{U}}$ & .08 & .09 & .33 & .28 & .18 \\
\hline${ }^{B_{G}}$ & .09 & .14 & .30 & .32 & .18 \\
\hline$c_{U}$ & .12 & .13 & .37 & .36 & .28 \\
\hline $\mathbf{c}_{\mathbf{G}}$ & .12 & .19 & .32 & .37 & .28 \\
\hline $\mathrm{qu}$ & .24 & .25 & .30 & .31 & .34 \\
\hline $\mathrm{D}_{\mathrm{G}}$ & .19 & .33 & .25 & .36 & .34 \\
\hline $\mathbf{E}_{\mathrm{U}}$ & .13 & .16 & .43 & .40 & .28 \\
\hline $\mathbf{E}_{G}$ & .14 & .22 & .37 & .44 & .28 \\
\hline $\mathbf{P}_{\mathrm{U}}$ & .08 & .30 & .09 & .27 & .20 \\
\hline $\mathbf{F}_{\mathrm{G}}$ & .08 & .15 & .08 & .10 & .20 \\
\hline
\end{tabular}

Predicted

The prescribed ordering appears to yield improvements which are in most cases as good as predicted, for elther unfform or gaussian distributions.

\section{Acknowledgment}

The authors wsh to thank Tom $\operatorname{col} 11 \mathrm{~ns}^{2}$ and Don Edwards for helpful conversations. In addition, they are grateful to Jonathan Schonfeld for pointing out the exdstence of the correlation term in Eq. (10).

\section{References}

1. See for example S. Ohnuma, AIP Donf. Proceeding No. 123 (1984), p. 415.

2. T.I. Collins (Fermilab-84/114) di scusses the need for five distortion parameters in a non-harmonlc Green's function type approach. He correctly points out the danger of manipulating a few "dominant" harmonica wthout worrying about a large number of nearby harmonics. As will be seen later, our echeme reduces all but a few harmonics at the same tine. 